\title{
UNUSUAL SITE HEMORRHAGIC COMPLICATIONS OF
}

\section{ANTICOAGULANTION}

\author{
A. Ashok Kumar
}

\section{ABSTRACT}

Anticoagulation has inherent quality to produce bleeding complications, more frequently in the elderly. Usual sites following anticoagulation is gastrointestinal, nasal, intracranial hemorrhage or petechial bleed. Here were reporting unusual sites of anticoagulation induced bleed. Rectus sheath hematoma is an unusual bleeding site, can mimic acute abdomen. Bleeding into intestinal wall can produce intussusception and presentation will be again as acute abdomen. Similarly, Sub lingual space is an unexpected site of bleeding and can present as upper airway obstruction or esophageal obstruction. Here we report one case of rectal sheath hematoma, 2 cases of sublingual hematoma and one case in the intestinal wall hematoma due to oral anticoagulation.

KEY WORDS: Hematoma, Bleeding.

\section{INTRODUCTION}

Anticoagulant therapy is a commonly prescribed treatment for most patients with both preventive and therapeutic indications and most frequently utilizing drugs in developing countries were oral Vitamin $\mathrm{K}$ antagonist. Oral anticoagulants work by depressing the activity of coagulation factors such as thrombin, prothrombin, and factors VII, IX, and X. There are several recognized indications for the use of oral anticoagulants. These include deep venous thrombosis, pulmonary embolism, mechanical valve replacement, cerebral venous thrombosis, certain hyper coaguable states, systemic embolism which is secondary to Atrial Fibrillation.

Hemorrhagic complications are to known to occur with anticoagulants use in day to day practice. One study following 3958 patients on warfarin reported an incidence of bleeding requiring hospitalization of 2.6 per 100 patient years [1]. Here we reporting unusual sites of hematoma (rectus sheath, subungual and in the intestinal wall).

\section{CASE 1}

An 80 year old male patient known case of Diabetes, hypertension, bronchial asthma , CAD underwent CABG 17 years back and chronic kidney disease on medical management. Now admitted with breathlessness since one week of NYHA class IV associated with orthopnea and paroxysmal nocturnal dyspnea since one week. At admission on general physical examination he had bilateral pedal edema and elevated JVP and vital parameters were stable with bilateral wheeze and audible S3. ECG suggestive of old IWMI, and $\mathrm{T}$ wave inversions in Leads $\mathrm{V} 1$ to V6. 2DECHO showed global hyperkinesia of Left ventricle with severe LV dysfunction with LV clot. Initially received heparin along with decongestive and vasodilator therapy for heart failure. On sixth day of his admission he was complaining of abdominal pain in right lower abdomen with tenderness in the right iliac fossa and with radiation to the peri umbilical region. Initial provisional diagnosis was acute appendicitis was made and surgical consultation was taken, advised conservative management with intravenous antibiotics and analgesics. He denied any history of trauma. Despite of analgesics his pain not decreasing and he noticed blackish discoloration in the peri umbilical region. Ultrasound abdomen did not reveal any abnormality. APTT was twice the normal range. Plain $\mathrm{CT}$ abdomen was done in view of raised serum creatinine and CKD and it was suggestive of a $46 \times 74 \times 122$ heterogeneous hyper density with average $\mathrm{HU}$ of 55 noted in the right rectus muscle in infra umbilical region (Fig 1).

${ }^{1}$ Senior resident, Departments of Cardiology, NIMS, India

Corresponding author: A Ashok Kumar

Email: ashokari@gmail.com 
Fig 1: CT Abdomen showing rectum sheath hematoma
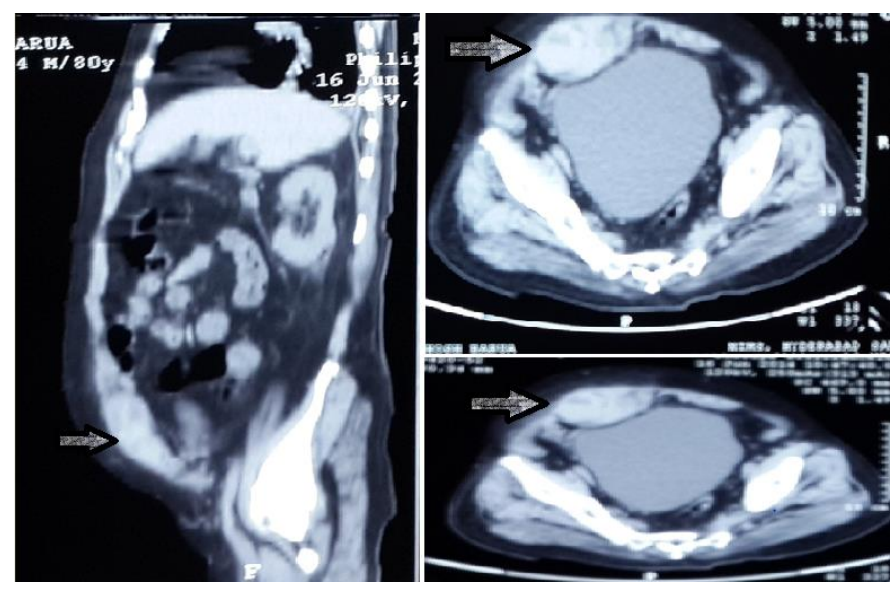

We stopped heparin and antiplatelets and his pain subsided within 24 hours and symptomatically improved.

\section{CASE 2:}

A 50 year old male patient known case of chronic rheumatic heart disease with severe mitral stenosis and moderate aortic regurgitation underwent double valve replacement with mechanical mitral and aortic valve replacement 4 years back. He also had atrial fibrillation and severe left ventricular dysfunction with previous history of left atrial appendage clot and he was on regular oral anticoagulants (Acenocoumarol $2 \mathrm{mg} / 3 \mathrm{mg}$ alternate day) use and on oral amiodarone for severe LV dysfunction and atrial fibrillation. Now he came with bleeding gums and swelling in the submandibular region since 3 days .

At admission he had pallor and his vital were stable. On local examination he had active bleeding from gums and submandibular and sublingual hematoma (Fig 2). His 2 D ECHO examination suggestive of dilated all four chambers and global hypokinesia of left ventricle severe Left ventricular dysfunction and normally functioning prosthetic valves with acceptable gradients across the mechanical prosthetic valves. His investigations revealed prothrombin time of 100 milliseconds and his INR (International normalized ratio) was 11, Renal parameters and platelet counts were normal with hemoglobin 10.2 grams. Oral anticoagulants were stopped and treated with injectable vitamin $\mathrm{K}$ single dose his bleeding gums has stopped and his INR was decreased to the therauptic range and restated with oral anticoagulants at a lower dose discharged in a stable condition form the hospital.

Fig 2: Sub-ungal haematoma.

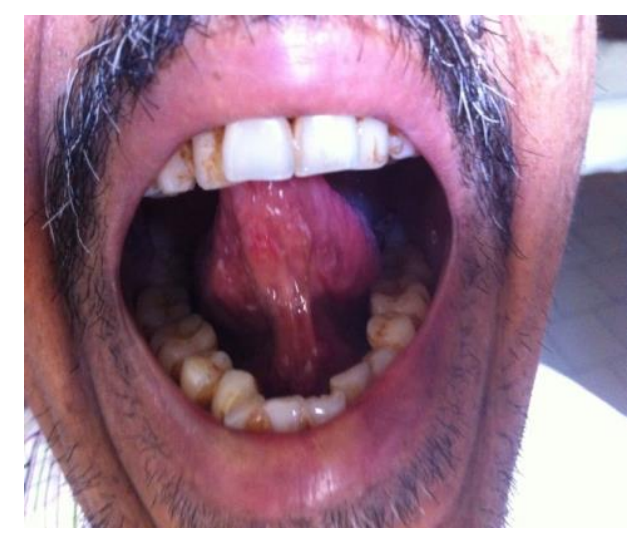

\section{CASE 3:}

A 46 year old female with past history Deep venous thrombosis and Pulmonary embolism 12 years back and diabetic since 4 years and Hypertensive since 4 years She was admitted with pain and swelling of right lower limb for 2 days one month back and diagnosed as deep venous thrombosis of right external iliac vein. Shortness of breath of one day duration. Computerized tomography pulmonary angiogram was done which suggestive of Acute Pulmonary embolism. She was treated with Low molecular weight Heparin for 1 week with overlap of oral anticoagulation with warfarin $5 \mathrm{mg}$ with INR at time if discharge was 2. After 3weeks of discharge from hospital she came to hospital with difficulty in swallowing liquids and throat pain while swallowing solid foods. On examination of the oral cavity she had a large, soft, dark red swelling involving the anterior region of the floor of her mouth, indicative of sublingual hematoma and the tongue was displaced anteriorly. There was no stridor or any other sign of major air way obstruction. The patients INR was 3.2 during second admission and warfarin was stopped and treated with single dose of intravenous vitamin $\mathrm{K}$.

Gradually the swelling has subsided and her symptoms improved over 1 week without any surgical intervention. Our patient was discharged on $13^{\text {th }}$ day of admission and INR was maintained at 2 with warfarin dose of $4 \mathrm{mg}$. At review one month later she had no signs of any bleeding manifestations. 
Fig 3: A. Sublingual Hematoma \& B Submandibular swelling

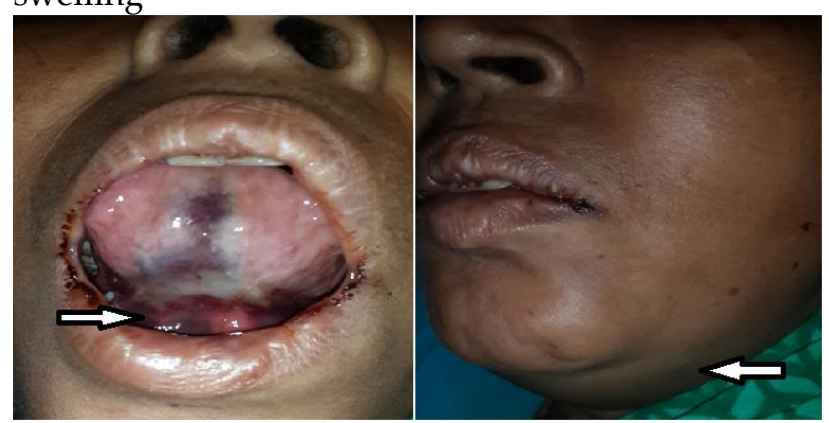

A

B

\section{CASE 4:}

45 year old male patient admitted with right SFV thrombosis with submissive pulmonary embolism. Patient received retrieval IVC filter from the left femoral vein along with heparin. At the time of implantation itself there was mild tilting of the filter. Patient was kept on Acitrom $2 \mathrm{mg}$ and adjusted the dose of Acitrom according to INR subsequently. After 4 months ha came with acute abdomen with significant fall of hemoglobin with 11 INR, but hemodynamically stable condition. Ct abdomen (Fig 4) showed pericaval hematoma with one strut of IVC filter protrusion and additional intestinal wall hematoma with intussusception.

Fig 4: CT abdomen showing titled IVC filter and intussusception.
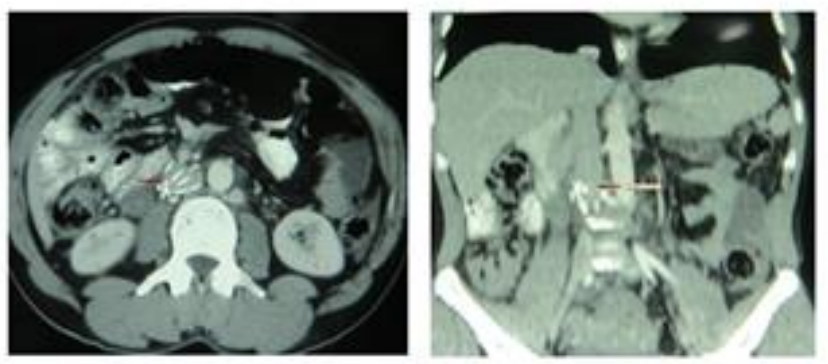

Patient received three packed cell transfusion and conservative management of intussusception with stoppage of anticoagulation. Patient discharged in stable condition.

\section{DISCUSSION}

Rectus sheath hematoma (RSH) results from damage to the superior or inferior epigastric arteries or their branches or from direct tear of the rectus abdominis muscle. Rectus sheath hematoma may results from excessively vigorous contractions of the rectus abdominis muscle often seen in strenuous exercise or repeated Valsalva with severe coughing, vomiting or straining at stool. Anticoagulation, especially heparin injection, has been described as an important etiological factor [1].

RSH can mimic any acute abdominal condition and consequently has a high rate of misdiagnosis [2]. One study found an incidence of RSH of $1.8 \%$ among 1257 patients admitted to hospital with abdominal pain [3]. Abdominal pain is the most common symptom but anorexia, nausea, vomiting, diarrhea, constipation, tenesmus, and bladder irritability can also manifest. Abdominal examination usually reveals a palpable, painful, firm, non-pulsatile mass corresponding to the rectus sheath. Ultrasound and CT scanning are commonly used to help confirm the diagnosis [4] Conservative management with rest, analgesia, compression, ice packs, reversal of anticoagulation, and transfusion is successful in the majority of cases [5]. Definitive management with angiography and embolization of the bleeding vessel or open surgery with clot evacuation, ligation of the bleeding vessel and closed-suction drainage should be considered in selected patients who fail or are unsuitable for conservative management. This includes those with enlarging hematomata, hemodynamic instability despite resuscitation, signs of peritonitis, inadequate pain control or persistent gastrointestinal or urinary symptoms. An index of high suspicion and early diagnosis is mandatory to make the correct diagnosis and avoid unnecessary exploratory laparotomy.

The sublingual space is an extremely rare site for bleeding, but this constitutes a life-threatening complication, if it is associated with signs and symptoms of airway obstruction [6]. In our case there were no signs of airway obstruction. The surgical drainage usually is not recommended. It is recommended that immediate reversal of the anticoagulation therapy, preferably with fresh frozen plasma but in our case as the patient had two mechanical prosthetic valves we have treated with vitamin $\mathrm{k}$.

Hemorrhages caused by a warfarin overdose usually appear in the genitourinary, gastrointestinal, retroperitoneal and intracranial areas and few reports have implied warfarin therapy as a cause of hematoma 
of the upper airway [6,7]. In our 2 cases sublingual hematoma is due to oral anticoagulation and in both cases the hematomas was noted spontaneously without any precipitating cause for bleeding like trauma.

Treatment of acute sublingual hematoma depends on the size of the hematoma and any compressive symptoms over upper respiratory tract or upper esophagus. Immediate identification of any reversible causes like reversal of anticoagulation ideally with fresh frozen plasma. But in our 2 cases of Sublingual hematoma both cases of excessive anticoagulation was reversed with Vitamin $\mathrm{K}$ and conservative management of the hematomas [7].

\section{CONCLUSION:}

Spontaneous Rectus sheath hematoma can be an unusual complication of the anticoagulation and it can mimic acute abdomen, accurate identification and appropriate reversal of the excess anticoagulation avoids unnecessary surgical explorations . Intestinal intussusception due to intestinal hematoma can be managed conservatively.

Sublingual hematomas can quickly develop into a lifethreatening condition, and early recognition, prompt medical treatment and continuous clinical monitoring may prevent the need for a surgical airway procedures.

\section{REFERENCES:}

1. Hollowell J, Ruigomez A, Johansson S, Wallander MA, Garcia-Rodriguez LA. The incidence of bleeding complications associated with warfarin treatment in general practice in the United Kingdom. British Journal of General Practice. 2003; 53:312-314.

2. J.E.F. Fitzgerald, 1.a. Fitzgerald, F.E. Anderson, A.G. Acheson. The changing nature of rectus sheath hematoma: case series and literature review. International journal of surgery. 2009;7: 2,150-154.

3. Klingler pj1, wetscher g, glaser $k$, tschmelitsch j, schmid $t$, hinder ra. The use of ultrasound to differentiate rectus sheath hematoma from other acute abdominal disorders. Surg endosc. 1999;13(11):1129-34.
4. Venkata M Alla, Showri M. Karnam, Manu Kaushik, and Joann Porter. Spontaneous rectus sheath hematoma. West j emerg med. 2010; 11(1): 76-79.

5. Mutlu Ünver, Murat Güner, Şafak Öztürk, Mustafa Ölmez, Yusuf Kumkumoğlu , Eyüp Kebabçi, Varlik Erol, Osman Bozbiyik, Nihat Zalluhoğlu,Cengiz Aydin, Gökhan Akbulut. Management of spontaneous rectus sheath hematoma: experience of double center. Acta medica mediterranea, 2014, 30: 811

6. Lim M, Chaudhari M, Devesa PM, Waddell A et al. Management of upper airway obstruction secondary to warfarin therapy: the conservative approach. J Laryngol Otol. 2006;120(2) .

7. Gonzalez - Garcia R, Schoendorff G, MunozGuerra Mf, et al. Upper airway obstruction by sublingual hematoma: a complication of anticoagulation therapy with acenocoumarol. Am J Otolaryngol. 2006;27:129-132. 